\title{
Benign metastasizing leiomyoma of the lung - case report and brief review of the literature
}

\author{
Vlad-Adrian Afrăsânie ${ }^{*, 1}$, Mariana lacob1, Dan Ferariu², Irina Demşa ${ }^{3}$, Lucian \\ Miron $^{3,4}$, Dana Elena Clement ${ }^{1}$
}

${ }^{1}$ Medical Oncology Department, Regional Institute of Oncology, Iaşi, Romania, ${ }^{2}$ Pathology Department, Regional Institute of Oncology, Iaşi, Romania, ${ }^{3}$ "Grigore T. Popa" University of Medicine and Pharmacy, Iaşi, Romania, ${ }^{4}$ Oncology Department, Regional Institute of Oncology, laşi, Romania

\begin{abstract}
Benign metastasizing leiomyoma is a rarely described disease characterized by extrauterine leiomyomatous lesions seen in premenopausal women with a history of uterine leiomyoma. Here, we present the case of a 47 year-old female who has undergone total hysterectomy for uterine leiomyoma at the age of 35. In 2012, eleven years after the hysterectomy, multiple pulmonary nodular lesions and a left ovary cyst were incidentally discovered during routine medical investigations. She underwent left salpingo-oophorectomy and resection of the pulmonary metastases. The histological and immunohistochemical findings led to the diagnosis of benign metastasizing leiomyoma. During 3 years of observation after the diagnosis was established, she presented with progressive disease, although she was under medroxyprogesterone acetate therapy. The therapy was substituted with aromatase inhibitors and the disease became stable. We present this case due to the rarity of this condition and also because it is a rare clinical presentation with an interesting therapeutic strategy.
\end{abstract}

Keywords: metastasizing leiomyoma, hormonal therapy, asymptomatic evolution

\section{Introduction}

Metastasizing is a hallmark of cancer, but there are some tumors like benign metastasizing leiomyoma (BML) that behave like a low-grade malignant neoplasm, although they have most of the benign characteristics.

BML is a rare entity, firstly described in 1939 by Steiner and typically diagnosed in premenopausal women with a history of hysterectomy for uterine leiomyoma [1]. The condition is characterized by benign spindle cell tumoral lesions proliferating from smooth

Received: October 2015; Accepted after review: December 2015; Published: December 2015

*Corresponding author: Vlad-Adrian Afrăsânie, Medical Oncology Department, lasi Regional Oncology Institute, 2-4, Gen. Henri Mathias Berthelot Street, 700483 lasi, Romania

Email: vlad afrasanie@yahoo.com muscles. The lungs are the most common site of metastasis, but other extrapulmonary sites like the lymph nodes, the deep soft tissue, the bones, the mesentery, the central nervous system and the heart have been identified as locations for the process of metastasizing as well [2]. Usually, the lesions growth is strongly associated with sex hormone levelsprogesterone, estrogen- and they usually have an asymptomatic course. The diagnosis can be confirmed from 3 months to 26 years after hysterectomy. Standardized treatment is not available and a careful follow-up is important, considering the low-grade malignant clinical behavior of the lesions. Treatment can include pulmonary nodule resection associated with hormonal therapy.

About 120 cases have been described in the literature, most of them involving the lungs as common site of metastasis [3-6]. We 
present a brief review of the literature along with a report of a rare case of pulmonary BML.

\section{Case report}

A 44 year-old female was evaluated in February 2012 in our hospital for imagistic abnormalities discovered incidentally on a pelvic ultrasound scan at another hospital one week earlier. The patient was asymptomatic at that moment. Her medical history revealed hypothyroidism at the age of 30 , treated with levothyroxine and total hysterectomy at the age of 35 for uterine leiomyoma. Physical examination revealed decreased breath sounds in the middle and lower fields of both lungs. The performance status (PS) at presentation was $0-1$ on ECOG scale. The results of the routine blood, urine and stool tests were normal. Ultrasound scan was repeated and showed a hypoechoic mass on the left ovary with irregular walls, thick septa and low impedance flow.

Suspecting a malignant tumor, the surgical team decided to operate the patient. Preoperative tests included a chest X-ray which noted multiple opaque nodular lesions ranging from $10 \mathrm{~mm}$ to $40 \mathrm{~mm}$ in the middle and lower fields of both lungs (Figure 1).

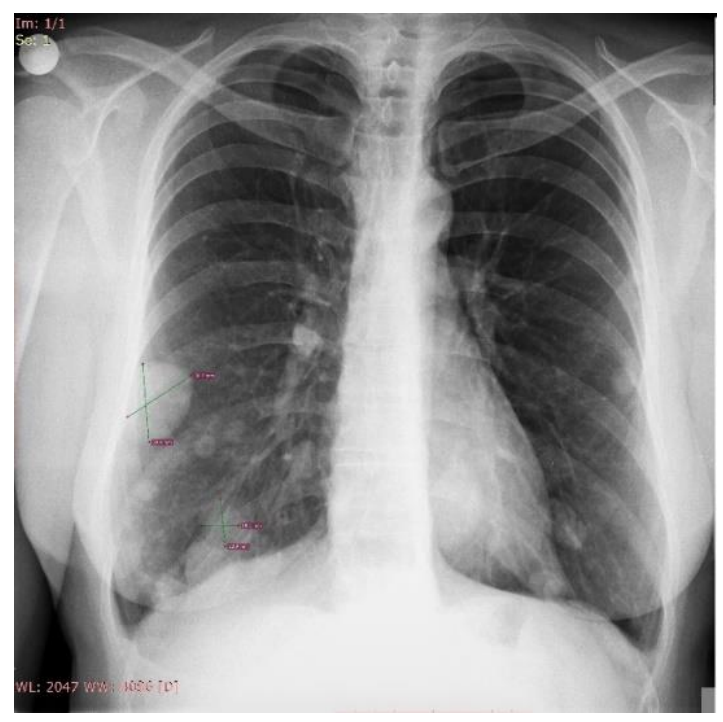

Fig.1. Chest X-ray: multiple opaque nodular lesions
The patient underwent left salpingooophorectomy and intravenous leiomyoma was diagnosed after the histopathologic examination (Figure 2).

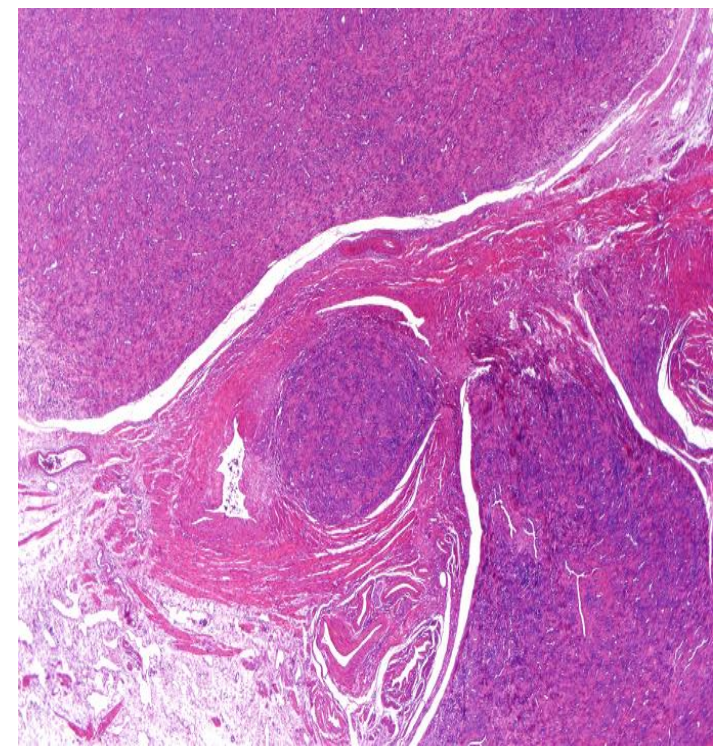

Fig. 2. Leiomyoma, intravenous growth, (H\&E, x 25)

After two weeks she was admitted to Thoracic Surgery Department, where a right thoracotomy with the excision of four tumors was performed.

Pathological macroscopic examination described four tumors of $5,5 / 3,5 / 2,5 \mathrm{~cm}$; $3,5 / 3 / 2 \mathrm{~cm} ; 3,5 / 2,4 / 1,5 \mathrm{~cm}$ and $3,52 / 2,1 \mathrm{~cm}$. Histological examination of the pulmonary fragments revealed multiple nodules composed of spindle cells, which indicated smooth muscle differentiation. Mitoses were very rare (Figure 3). Immunohistochemistry revealed that the tumor cells were diffusely positive for desmin and smooth muscle actin (Figure 4).

Proliferative marker (Ki-67) was below 5\% in the tumor cells (Figure 5). Immunohistochemical staining for estrogen and progesterone receptors revealed strong positivity in both of the stains (Figure 6). These histopathologic findings, established the final diagnosis of benign metastasizing leiomyoma. 


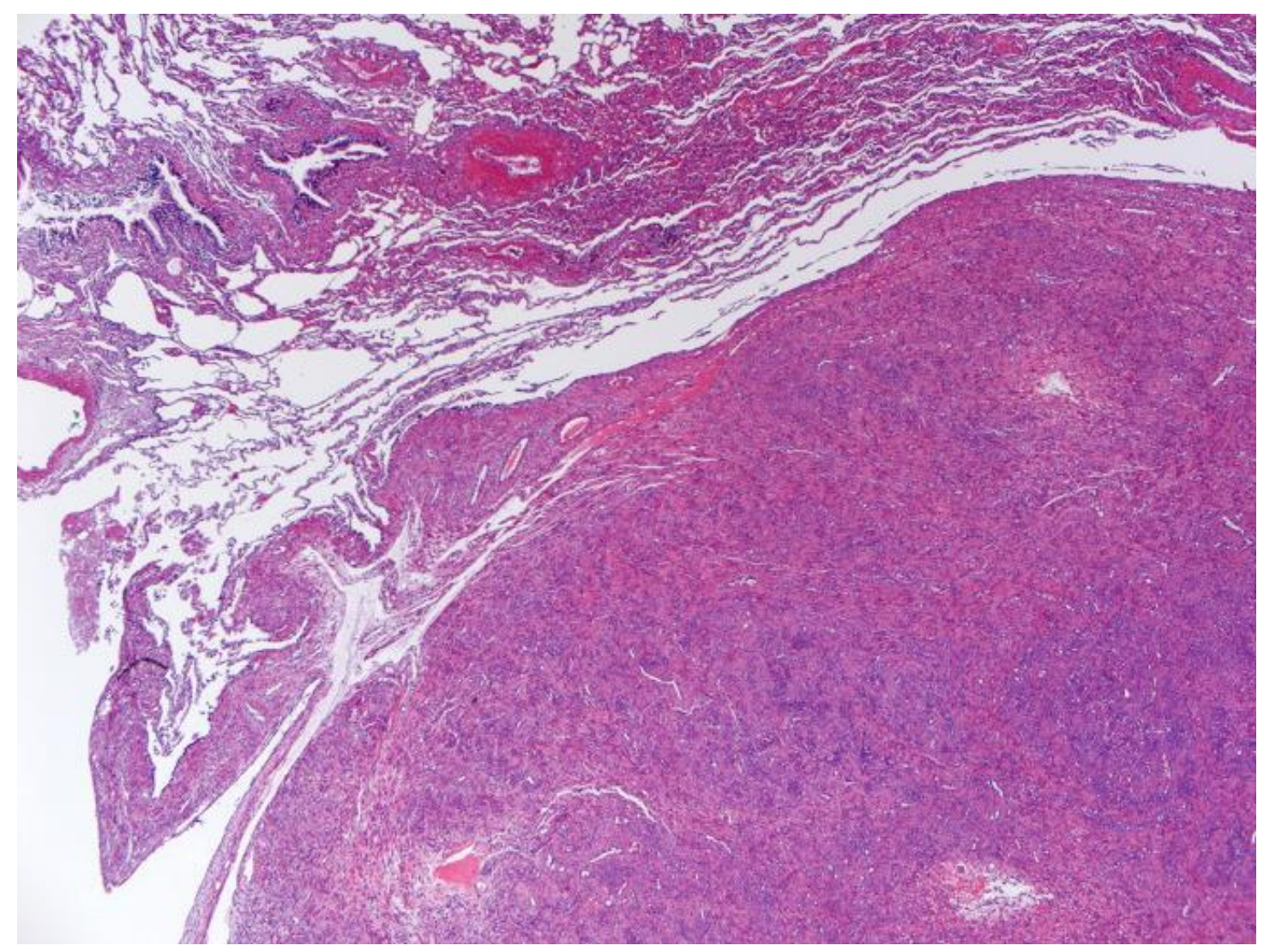

Fig. 3. Metastasizing leiomyoma, lung tissue (top) (H\&E, x25)

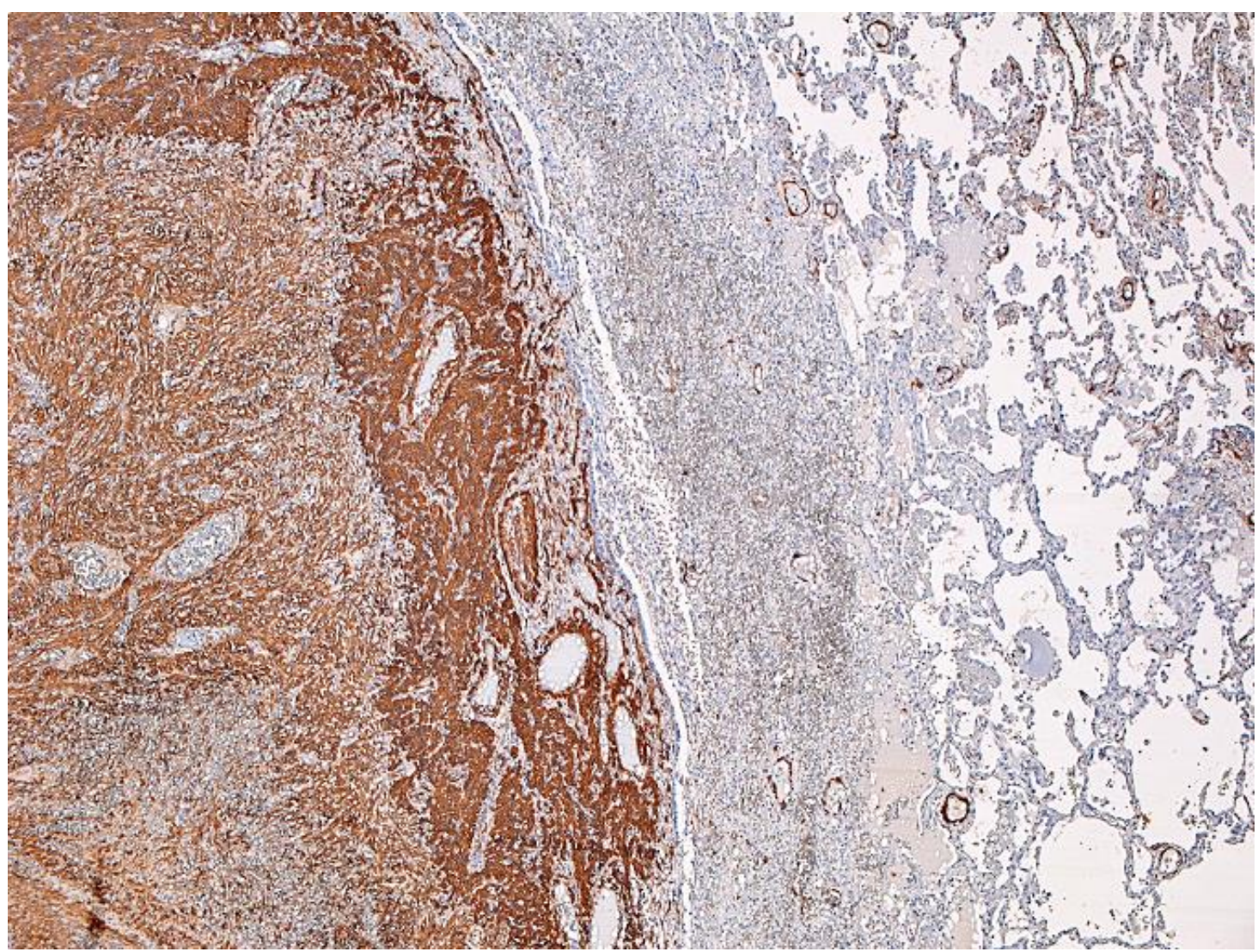

Fig.4. Smooth muscle actin, diffusely positive in tumor cells (left), lung tissue (right). (IHC, Ab anti-SMA, x25) 


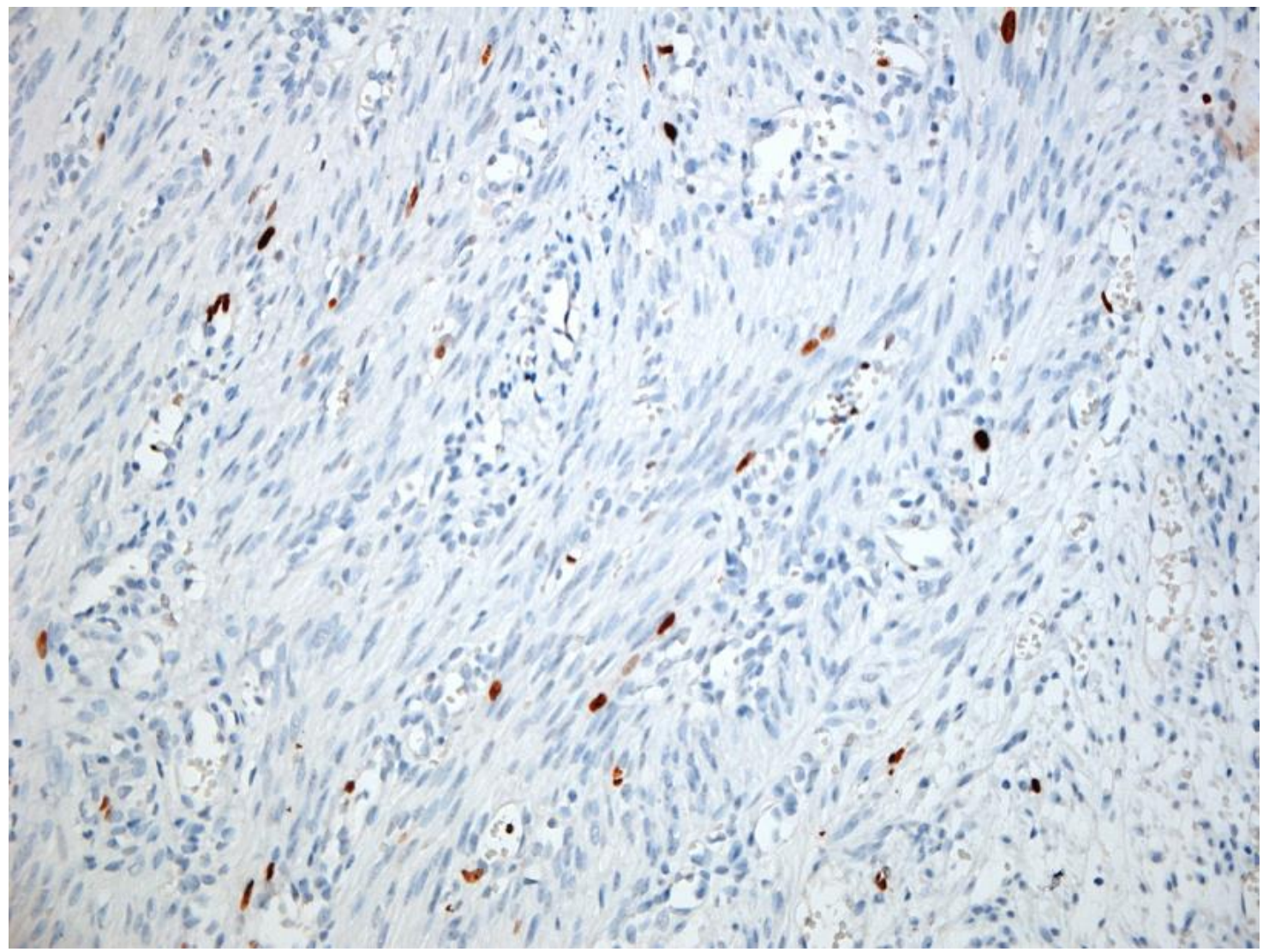

Fig. 5. Ki67 positive in rare cells (IHC, Ab anti-Ki67, x200)

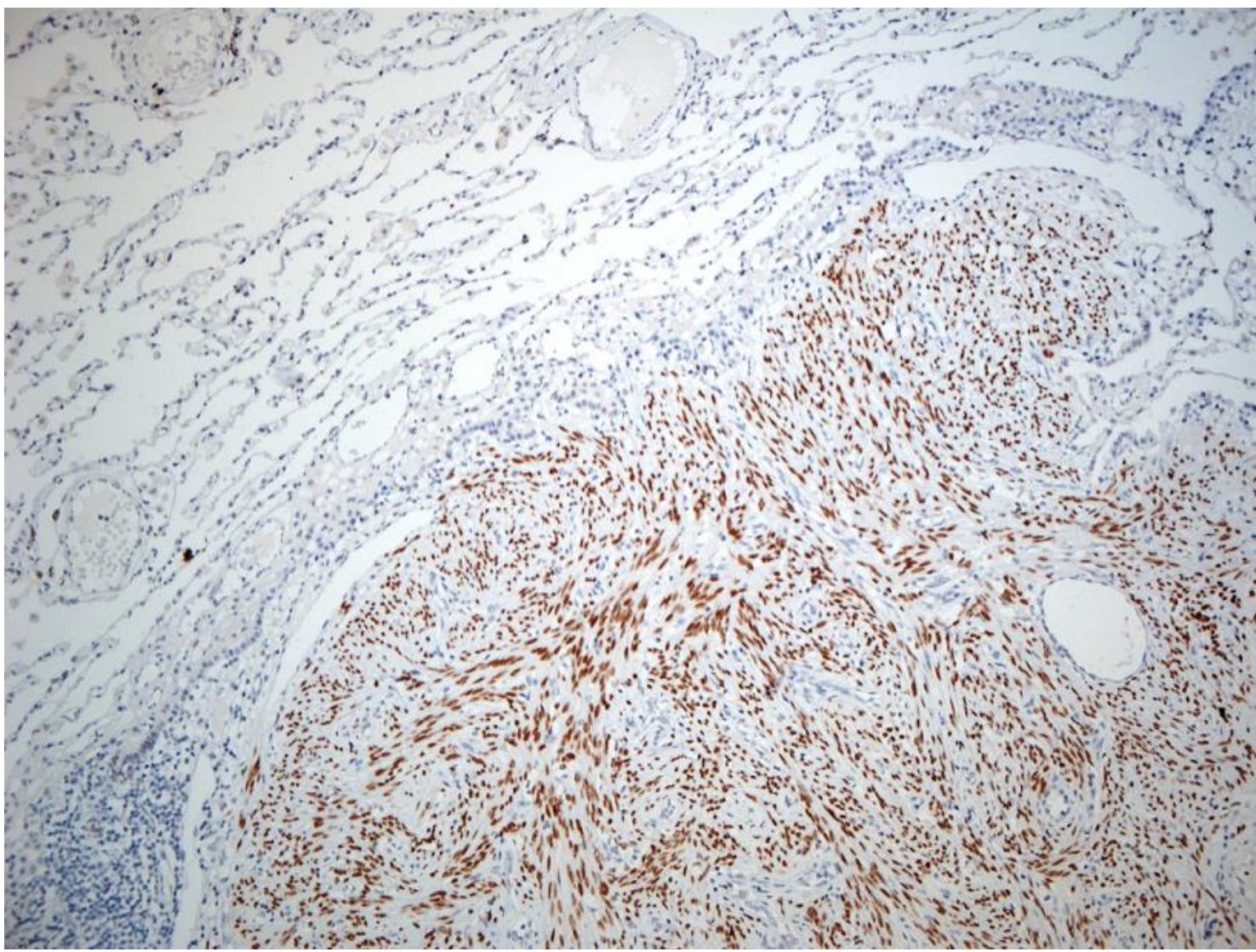

Fig. 6. Estrogen receptor diffusely positive (IHC, Ab anti-EgR, x100) 
In October 2012 the patient was admitted in the Medical Oncology Department. She was asymptomatic, despite the fact that a thoracic CT scan showed multiple round, contrast enhancing, well-circumscribed, noncalcified nodules, which were located in the left lower lobe and in the right medial lobe, ranging from $9 \mathrm{~mm}$ to $27 \mathrm{~mm}$ (Figure 7 ).

The patient was treated with progesterone receptor agonist: medroxiprogesteron acetate $160 \mathrm{mg}$ tablets, orally, twice a day. The treatment was well tolerated, without side effects. However, after four months, in February 2013, medroxiprogesteron acetate

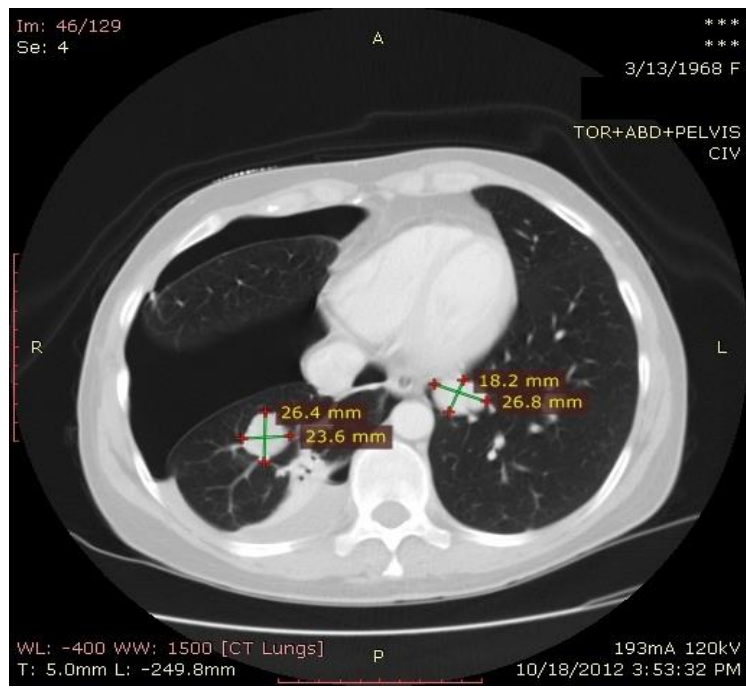

Fig. 7. Dimensions of target lesions on thoracic CT scan in October 2012

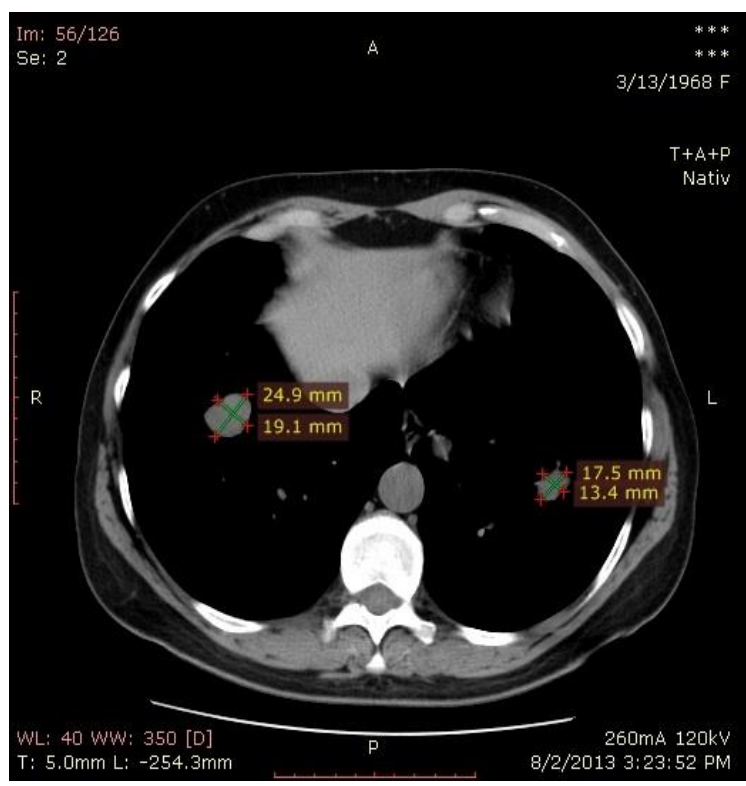

Fig. 9. Dimensions of target lesions on thoracic CT scan in August 2013 was substituted with aromatase inhibitor anastrozole at a dose of $1 \mathrm{mg}$ daily, due to the fact that thoracic CT scan revealed progression of the disease (Figure 8).

The next three thoracic CT scans in August 2013, January 2014 and October 2014 showed stable disease according to response evaluation in solid tumors (RECIST) (Figures 9 and 10).

In the last six months, except for light fatigue, the patient was asymptomatic, without imagistic evidence of progressive disease. We decided to continue treatment with anastrozole and periodically survey the lung lesions.

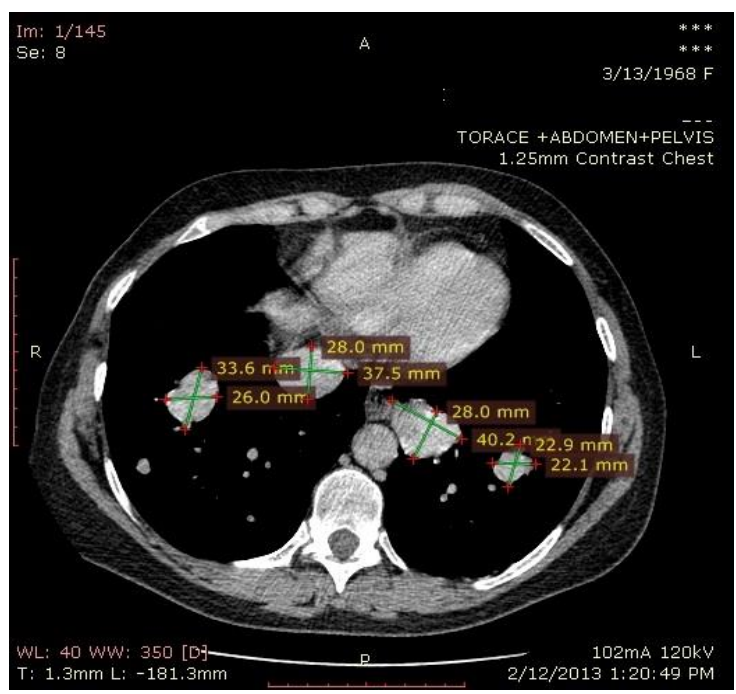

Fig. 8. Dimensions of target lesions on thoracic CT scan in February 2013

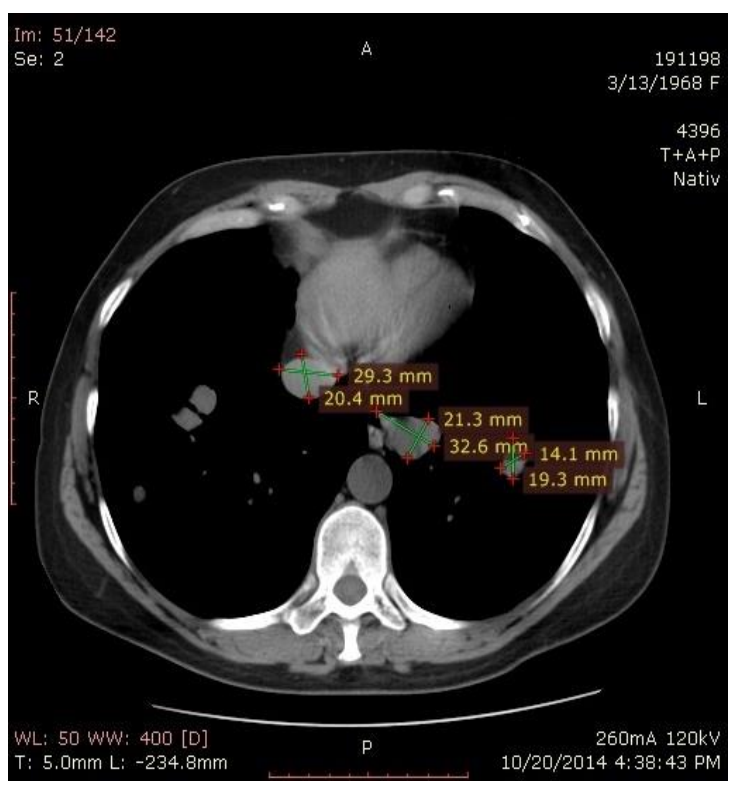

Fig. 10. Dimensions of target lesions on thoracic CT scan in October 2013 


\section{Discussions}

BML is an entity characterized by extrauterine leiomyomatous lesions without histological signs of malignancy, which clinically and radiologically simulate metastatic behavior. This rare condition is found in women of reproductive age with a history of uterine leiomyoma.

The first case was reported in 1889 by Krische, who described uterine fibromyomas metastasized to many parts of the body. Afterwards, in 1893, Langerhans reported the second case, that of a 60 year old woman with multiple nodules in the uterus and multiple pulmonary metastases. In 1901, Minkowski recorded the third case, that of a 43 year old woman with multiple nodules of smooth muscle in the lung and liver, 2 years after a complete hysterectomy for a fibromyoma of the uterus. The first to introduce the term "benign metastasizing fibroleiomyoma" was P.E. Steiner, in 1939, at the University of Chicago, reporting the case of a 36 year old woman who died after massive pulmonary and hilar lymphatic metastases of a uterine leiomyoma. He described it "as a tumor composed histologically, in both the primary growth and its metastasis, of benign appearing, fully differentiated smooth muscle cells and dense connective tissue". The term 'benign metastasizing leiomyoma' was formulated by J.P. Horstman to distinguish the myometrial origin of the disease $[5,7,8]$.

The pathogenesis of BML is controversial. There are several main hypotheses that have been proposed: (a) it could be a benign uterine tumor that spreads through hematic route to the lungs or other organs, since the majority of patients had prior myomectomy or hysterectomy; (b) it could be a low grade leiomyosarcoma metastasizing to lungs; (c) it could represent primary pulmonary leiomyomatosis too, but coexisting with uterine leiomyoma [5]. The theory of lymphovascular spread is one of the most widely accepted hypotheses and it is based upon reports of spontaneous regression of pulmonary leiomyoma during pregnancy [8]. There have been described consistent chromosomal aberrations, $19 q$ and $22 q$ terminal deletions, which suggested that the BML can be genetically determined. Almost $25 \%$ of the uterine leiomyomas may possess a balanced translocation, trisomy 12 and rearrangement of $6 p$ [9]. However these chromosomal abnormalities associated with BML have been difficult to characterize [9].

The pathological characteristics attest the benign phenotype of the tumor. Cytologic atypia, coagulative tumor cell necrosis, increased mitoses with a low $\mathrm{Ki}-67$ index and the absence of high cellularity indicate a low tumor cell proliferative state and the benign nature of these lesions. In two different studies the $\mathrm{Ki}-67$ expression was reported to be between $2.3 \%$ and $2.9 \%[1,10]$. Various immunohistochemical markers, such as vimentin, smooth muscle actin and desmin are present in $80 \%$ of cases, confirming the mesenchymal origins with smooth muscle differentiation of these tumors. BML expresses estrogen and progesterone receptors [11, 12]. In our case, histological examination of the pulmonary fragments revealed multiple nodules composed of spindle cells, which indicated smooth muscle differentiation and very rare mitoses. Immunohistochemistry revealed that the tumor cells were diffusely positive for desmin and smooth muscle actin, the Ki-67 proliferation index was approximately $5 \%$ and estrogen and progesterone receptors were positive.

The age distribution at diagnosis varies between 19 to 77 years with a mean age of 40-49 years. Characteristics of patients with $\mathrm{BML}$ are widely various, in spite of its benign cytology. All patients were reported to have a history of uterine leiomyoma and a prior curettage, myomectomy or hysterectomy and metastasizing occurs mainly in premenopausal and perimenopausal woman. In a review of 99 cases was found that 77 patients had undergone hysterectomy and 22 patients had undergone myomectomy [7]. The period between hysterectomy or myomectomy and the appearance of BML ranged from 3 months to 26 years [13]. However, there have been described cases in which the uterine tumor was discovered simultaneously or even after the metastases were found. Extrapulmonary lesions have been documented to appear in 
lymph nodes, deep soft tissue, omentum, mesentery, bone, skull base, spine and heart. There have also been reported two cases with breast metastasis [14, 15]. Our patient underwent hysterectomy for uterine leiomyoma at the age of 35 and the diagnosis of BML was established 9 years later, when she was 44-year old. Metastases were found in the lungs.

Because the disease has an indolent evolution, the patients with pulmonary metastases are often asymptomatic at presentation, the lesions being incidentally discovered on X-ray. Symptoms such as cough, chest pain, rare hemoptysis and dyspnea have been observed in some cases. However, there is little correlation between symptoms and radiological findings [6]. The survival time varies between 7 months and 101 months (median survival time of 94 months) after the surgical treatment of pulmonary metastases and it depends on the extension of the pulmonary nodules [16]. The disease course was followed in $24 \mathrm{BML}$ patients and it has been observed that $13 \%$ of the patients died within two years after the surgical intervention and $46 \%$ had survived more than four years. The longest survival period was 36 years in a case of one patient with extensive lung involvement [11]. Even though progression is usually slow, it finally leads to massive pulmonary invasion, with its consequent morbidity [17, 18]. The disease process is indolent in postmenopausal patients, while progressive respiratory failure and even death is more frequent in the premenopausal patients [11]. Light fatigue was the only symptom that our patient related in contrast with the imagistic findings. The disease evolution was indolent, slow progressive and then stable in the past three years.

Radiologically, BML appears as wellcircumscribed solitary or multiple nodules, with sizes ranging from a few millimeters to several centimeters, scattered within the normal interstitium. Endobronchial and pleural sparring is also characteristic of BML. Miliary dissemination, cavitary lesions or interstitial lung disease have rarely been observed. A radiologic evaluation reported multiple nodules in $87 \%$ of cases, $70 \%$ bilateral and $17 \%$ unilateral, with a solitary nodule found only in $13 \%$ of all patients [6]. In our case, the most common pattern of BML, with multiple nodules in both lungs, was present.

In the case of patients with a history of uterine leiomyoma, especially if they had undergone uterine tumor surgery, the possible diagnosis of BML should be considered when pulmonary nodules and diffuse lesions are present. Because the imaging features of BML are nonspecific, it is difficult to confirm this pathology without further investigations, therefore thoracoscopic lung biopsy or open lung biopsy with histopathologic and immunohistochemistry examination are mandatory and represent the standard method to establish the diagnosis of pulmonary BML [19].

The differential diagnosis includes: leiomyosarcoma, pulmonary metastases, pulmonary tuberculosis, pulmonary echinococcosis, primary pulmonary leiomyomatosis, lymphangioleiomyomatosis and fibroleiomyomatous hamartoma. The clinical characteristics described are specific and differ from those of the other diseases [20].

There are several treatment options for BML. The first method of treatment that doctors opt for is the surgical excision of the secondary lesions [19]. An evaluation of the patient's condition is highly indicated at regular time intervals following the surgery, in order to discover any recurrence of the metastasis.

As these tumors express estrogen and progesterone receptors, another treatment option is hormonal manipulation of the disease. Information about hormonotherapy are few and sometimes contradictory. A review of the literature reveals advancing efforts to control gonadal hormone secretion in patients with BML. Bilateral oophorectomy is effective in controlling tumor growth [21, 22]. However, the possibility of medical castration is preferred, due to its reversibility and its potential to allow symptoms control when surgical management is not possible, needs to be postponed or has been ineffective. The use of long-acting $\mathrm{GnRH}$ analogs, which suppress the endogenous gonadotropin secretion necessary for gonadal steroid production, has been described with good 
results in several reports [15, 18, 23-26]. In one case, GnRH agonists have suppressed the growth of the lung nodules, and no increase in size has been noticed for a period of 36 months [17]. Progesterone treatment has also been shown to be effective in both the prophylaxis against recurrences and the regression of the leiomyomatous tumors because of its ability to suppress the hypothalamic-pituitary-gonadal axis, thereby reducing ovarian estrogen synthesis [27-31]. The direct effect of progesterone on leiomyoma cells has been studied in cultures. Estradiol and progesterone increase the expression of the proliferating nuclear antigen, a marker for cell proliferation. Furthermore, estradiol but not progesterone augments proliferating nuclear cell antigen expression in cultures of normal myometrial cells. This may explain why in some BML patients, progestins were ineffective or caused worsening and further tumor spread [32, 33]. Estrogen receptor antagonists, such as tamoxifen are used to treat BML, but the information are few and contradictory. It has been published the case of a 47 year old woman who had been treated with tamoxifen and had a stable disease for about 1 year. On the contrary, another patient had been unsuccessfully treated with tamoxifen [6, 34]. Aromatase inhibitors have been used to treat BML. Anastrozole and other selective nonsteroidal inhibitors of aromatase-P450 enzyme reduce estradiol concentrations by acting on both the gonads, peripheral and tumor tissues. It has been reported in the literature a case of a 46year-old woman treated with oral anastrozole after total hysterectomy with bilateral salpingo-

\section{References}

1. Patton KT, Cheng L, Papavero V, et al. Benign metastasizing leiomyoma: clonality, telomere length and clinicopathologic analysis. Mod Pathol 2006; 19:130-140.

2. Taftaf $R$, Starnnes $S$, Wang J, et al. Benign metastasizing leiomyoma: A rare type of lung metastases - two case reports and review of the literature. Case Rep Oncol Med 2014; (6):842801.

3. Sehgal R, Maghrabi A. Benign metastasizing leiomyoma: 'a sheep in wolf's clothing'. Community oncology 2013; 10(4):122-124. oophorectomy and showed a stable disease for 15 months after operation. Some authors suggest that anastrozole in combination with raloxifene would have better outcomes in the treatment of BML because they have different mechanisms of action and would determine a synergistic effect [17]. The clinical evolution of patients with BML varies from a chronic asymptomatic course to a rapid progression, leading to respiratory failure and death. Therefore, an individual treatment strategy should be considered for each patient depending on the size and location of the tumor and the hormone receptor status [35]. In our case, the excision of the pulmonary tumors was performed at first and then the patient followed treatment with progesterone receptor agonist for four months, until progression of the disease was documented. After that, treatment with aromatase inhibitor, anastrozole, was started and the disease became stable.

\section{Conclusion}

$\mathrm{BML}$ is a rare tumor with benign histologic characteristics and the biological property to metastasize. The disease appears in women who have a personal history of surgical excision of uterine leiomyoma. The lungs are the most frequent site for metastases, which is why BML should be included in the differential diagnosis of solitary or multiple pulmonary nodules in these patients. There is no standard treatment, but surgical resection and/or antihormonal therapy are preferred.

4. Huang $\mathrm{H}$, Balos $\mathrm{L}$, Chen F. Pulmonary benign metastasizing leiomyoma appears 26 years after resection of uterine leiomyoma. $N A J$ Med Sci 2012; 5(1):55-57.

5. Fatima S, Ahmed Z, Azam M. Benign metastasizing leiomyoma. Indian $J$ Pathol Microbiol 2010; 53:802-4.

6. Abramson S, Gilkeson RC, Goldstein JD, Woodard PK, Eisenberg R, Abramson N. Benign metastasizing leiomyoma: clinical, imaging and pathologic correlation. $A m \mathrm{~J}$ Roentgenol 2001; 176(6):1409-1413. 
7. Mlika M, Ayadi-Kaddour A, Smati B, et al. Benign metastasizing leiomyoma: report of 2 cases and review of the literature. Pathologica 2009; 101(1), 9-11.

8. Ağaçkiran Y, Findik G, Üstün L, Aydoğdu K, Kaya S. Pulmonary benign metastasizing leiomyoma: an extremely rare case. Turkish $\mathrm{J}$ Pathol 2013; 1(1):1-4.

9. Jo JH, Lee JH, Kim DC, et al. A case of benign metastasizing leiomyoma with multiple metastastasis to the soft tissue, skeletal muscle, lung and breast. Korean J Intern Med 2006; 21(3):199-201.

10. Lim SY, Park JC, Bae JG, et al. Pulmonary and retroperitoneal benign metastasizing leiomyoma. Clin Exp Reprod Med 2011; 38(3):174-177.

11. Ki EY, Hwang SJ, Lee KH, Park JS, Hur SY. Benign metastasizing leiomyoma of the lung. World J Surg Oncol 2013; 11:279.

12. Koh DM, Burn PR, King DM. Benign metastasizing leiomyoma with intracaval leiomyomatosis. Br J Radiol 2000; 73:435-7.

13. Radzikowska E, Szczepulska-Wójcik E, Langfort R, Oniszh K, Wiatr E. Benign pulmonary metastasizing leiomyoma uteri. Case report and review of literature. Pneumonologia i alergologia polska 2012; 80(6):560-4.

14. $\mathrm{Ma} \mathrm{H}$, Cao J. Benign pulmonary metastasizing leiomyoma of the uterus: a case report. Oncol Lett 2015; 9(3): 1347-1350.

15. Alessi G, Lemmerling $M$, Vereecken $L$, De Waele L. Benign metastasizing leiomyoma to skull base and spine: a report of two cases. Clin Neurol Neurosurg 2003; 105:170-174.

16. Ahmad SZ, Anupama R, Vijaykumar DK. Benign metastasizing leiomyoma - case report and review of literature. Eur J Obstet Gynecol Reprod Biol 2011; 159:240-1.

17. Rivera JA, Christopoulos S, Small D, Trifiro M. Hormonal manipulation of benign metastasizing leiomyomas: report of a two cases and review of the literature. $J$ Clin Endocrinol Metab 2004; 89:3183-8.

18. Hague WM, Abdul Wahid NA, Jacobs HS, Graft I. Use of LHRH analogue to obtain reversible castration in a patient with benign metastasizing leiomyoma. $\mathrm{Br} J$ Obstet Gynaecol 1986; 93:455-60.

19. Chen S, Liu RM, Li Tian. Pulmonary benign metastasizing leiomyoma: a case report and literature review. J Thorac Dis 2014; 6(6): E92-E98.

20. Gomez FJT, Tristan AA, Olivera FJT. Benign metastasizing leiomyoma of the lung. Arch Bronconeumol 2007; 43(1): 43-52.
21. Evans AJ, Wiltshaw E, Koshanowski SJ, Macfarlane A, Sears RT. Metastasizing leiomyoma of the uterus and hormonal manipulations: case report. $\mathrm{Br} J$ Obstet Gynaecol 1986; 93:646-648.

22. Uchida $T$, Tokumaru $T$, Kojima $H$, Nakagawaji $\mathrm{K}$, Imaizumi M, Abe T. A case of multiple leiomyomatous lesions of the lung: an analysis of flow cytometry and hormone receptors. Surg Today 1992; 22:265-268.

23. Nakanishi $S$, Nakano $K$, Hiramoto $T$, Shimizu M, Nakamura K, Yamane N. So-called benign metastasizing leiomyoma of the lung presenting with bone metastases. Nihon Kokyuki Gakkai Zasshi 1999; 37:146-150.

24. Maheux R, Samson $Y$, Farid NR, Parent JG, Jean $C$. Utilization of luteinizing hormonereleasing hormone agonist in pulmonary leiomyomatosis. Fertil Steril 1987; 48:315-317.

25. Sabatini R, Ferreri R, Distante G, Loizzi V, Loizzi P. Benign metastasizing leiomyoma in the lung: a case report. Eur $J$ Gynaecol Oncol 2002; 23:445-446.

26. Nardo LG, lyer L, Reginald PW. Benign pulmonary metastasizing leiomyomatosis in pregnancy: a rare complication after cesarean section. Acta Obstet Gynecol Scand 2003; 82:770-772.

27. Perel E, Daniilescu D, Kharlip L, Blackstein M, Killinger DW. Steroid modulation of aromatase activity in human cultured breast carcinoma cells. J Steroid Biochem 1988; 29:393-399.

28. Jautzke G, Müller-Ruchholtz E, Thalmann U. Immunohistological detection of estrogen and progesterone receptors in multiple and well differentiated leiomyomatous lung tumors in women with uterine leiomyomas (so-called benign metastasizing leiomyomas). A report on 5 cases. Pathol Res Pract 1996; 192:215223.

29. Cramer SF, Meyer JS, Kraner JF, Camel M, Mazu MT, Tenenbaum MS. Metastasizing leiomyoma of the uterus. S-phase fraction, estrogen receptor and ultrastructure. Cancer 1980; 45:932-937.

30. Maruo T, Matsuo H, Samoto T, et al. Effects of progesterone on uterine leiomyoma growth and apoptosis. Steroids 2000; 65:585-592.

31. Pawlik C, Wildberger JE, Tietze L, Matern S, Busch N. Benign metastasizing leiomyoma of the lung-a rare differential diagnosis of pulmonary space-occupying lesions. Dtsch Med Wochenschr 2001; 126:551-555.

32. Wentling GK, Sevin BU, Geiger XJ, Bridges MD. Benign metastasizing leiomyoma responsive to megestrol: case report and review of the literature. Int $J$ Gynecol Cancer 2005; 15:1213-1217. 
33. Beck MM, Biswas B, D'Souza A, Kumar R. Benign metastasizing leiomyoma after hysterectomy and bilateral salpingooophorectomy. Hong Kong Med J 2012; 18(2):153-155.

34. Säynäjäkangas $O$, Maiche $A G$, Liakka KA. Multiple progressive pulmonary leiomyomatous metastases treated with tamoxifen: a case report with a review of the literature. Acta Oncol 2004; 43(1):113-114.

35. Nasu K, Tsuno A, Takai N, Narahara H. A case of benign metastasizing leiomyoma treated by surgical castration followed by an aromatase inhibitor, anastrozole. Arch Gynecol Obstet 2009; 279(2):255-257. 